\title{
NEW CHARACTERIZATIONS OF BLOCH SPACES, BERS-TYPE AND ZYGMUND-TYPE SPACES AND RELATED QUESTIONS
}

\author{
M. GARAYEV, H. GUEDIRI, H. SADRAOUI
}

\begin{abstract}
In terms of Berezin symbols, we give new characterizations of the Bloch spaces $\mathcal{B}$ and $\mathcal{B}_{0}$, Bers-type and the Zygmund-type spaces of analytic functions on the unit disc $\mathbb{D}$ in the complex plane $\mathbb{C}$. We discuss some properties of Toeplitz operators on the Bergman space $L_{a}^{2}(\mathbb{D})$. We provide a new characterization of certain function space with variable exponents. Namely, given a function $f(z)=\sum_{n=0}^{\infty} \widehat{f}(n) z^{n} \in \operatorname{Hol}(\mathbb{D})$ with a bounded sequence $\{\widehat{f}(n)\}_{n \geqslant 0}$ of Taylor coefficients $\widehat{f}(n)=\frac{f^{(n)}(0)}{n !},(n=0,1,2, \ldots)$, we have $f \in H_{p(\cdot), q(\cdot), \gamma(\cdot)}$
\end{abstract} if and only if

$$
\int_{0}^{1}\left(\frac{1}{2 \pi} \int_{0}^{2 \pi}\left|\widetilde{D}_{\left(\widehat{f}(n) e^{i n t}\right)}(\sqrt{r})\right|^{p(t)} d t\right)^{\frac{q(t)}{p(t)}}(1-r)^{\frac{\gamma(t) p(t)-q(t)}{p(t)}} d r<+\infty .
$$

Here $D_{\left(a_{n}\right)}$ denotes the associate diagonal operator on the Hardy-Hilbert space $H^{2}$ defined by the formula $D_{\left(a_{n}\right)} z^{n}=a_{n} z^{n}(n=0,1,2, \ldots)$.

Keywords: Bers-type space, Zygmund-type space, Bloch spaces, Berezin symbol.

Mathematics Subject Classification: 47B33, 30H30

\section{INTRODUCTION}

Let $\mathbb{D}$ be the unit disc in complex plane $\mathbb{C}, \mathbb{D}=\{z \in \mathbb{C}:|z|<1\}$ and let $\operatorname{Hol}(\mathbb{D})$ be the class of functions analytic in $\mathbb{D}$. We denote by $H^{\infty}=H^{\infty}(\mathbb{D})$ the space of bounded analytic functions on $\mathbb{D}$. Recall that a function $f \in \operatorname{Hol}(\mathbb{D})$ belongs to the Bloch space $\mathcal{B}=\mathcal{B}(\mathbb{D})$ if

$$
\|f\|_{b}:=\sup _{z \in \mathbb{D}}\left(1-|z|^{2}\right)\left|f^{\prime}(z)\right|<+\infty \text {. }
$$

Being equipped with the norm $\|f\|_{\mathcal{B}}=|f(0)|+\|f\|_{b}, \mathcal{B}$ is a Banach space. Let $\mathcal{B}_{0}=\mathcal{B}_{0}(\mathbb{D})$ be the space consisting of all $f \in \mathcal{B}$ satisfying

$$
\lim _{|z| \rightarrow 1}\left(1-|z|^{2}\right)\left|f^{\prime}(z)\right|=0 .
$$

This space is called a little Bloch space.

M. Garayev, H. Guediri, H. Sadraoui, New Characterizations of Bloch spaces, Bers-type And Zygmund-Type SPACES And Related Questions.

(c) Garayev M., Guediri H., Sadroui H. 2018.

The authors would like to extend their sincere appreciation to the Deanship of Scientific Research at King Saud University for its funding of this research through the Research Group Project no RGP-VPP-323.

Submitted June 29, 2017. 
Let $\alpha \geqslant 0$. The Bers-type space, denoted by $H_{\alpha}^{\infty}=H_{\alpha}^{\infty}(\mathbb{D})$, is a Banach space consisting of all $f \in \operatorname{Hol}(\mathbb{D})$ such that

$$
\|f\|_{\infty, \alpha}=\sup _{z \in \mathbb{D}}\left(1-|z|^{2}\right)^{\alpha}|f(z)|<+\infty .
$$

Clearly, $H_{0}^{\infty}=H^{\infty}$.

Let $0<p, q<+\infty, \gamma>-1$. If a function $f \in \operatorname{Hol}(\mathbb{D})$ is such that

$$
\|f\|_{H p, q, \gamma}^{q}:=\int_{0}^{1}\left(\frac{1}{2 \pi} \int_{0}^{2 \pi}\left|f\left(r e^{i \theta}\right)\right|^{p} d \theta\right)^{\frac{q}{p}}(1-r)^{\gamma} d r<+\infty
$$

we say that $f$ belongs to a mixed norm space denoted by $H_{p, q, \gamma}=H_{p, q, \gamma}(\mathbb{D})$.

Let $\beta>0$. The Zygmund-type space denoted by $\mathcal{Z}^{\beta}$ consist of all $f \in \operatorname{Hol}(\mathbb{D})$ obeying

$$
\|f\|_{\mathcal{Z}^{\beta}}:=|f(0)|+\left|f^{\prime}(0)\right|+\sup _{z \in \mathbb{D}}\left(1-|z|^{2}\right)^{\beta}\left|f^{\prime \prime}(z)\right|<+\infty .
$$

The space $\mathcal{Z}^{\beta}$ becomes a Banach space with the above norm $\|\cdot\|_{\mathcal{Z}^{\beta}}$. Let $\beta=1$. Then $\mathcal{Z}^{1}=\mathcal{Z}$ is the classical Zygmund space. For more information on the Bers-type, Zygmund-type and Bloch spaces on the unit disc $\mathbb{D}$, see, e.g., K. Zhu [14, 15], X. Zhu [16], S. Stević [11], [12], Y. Ren [9], P. Duren [2], J. Shi [10].

Recall that the following old problem for most of functional spaces $X$ of analytic functions in $\mathbb{D}$, including the Hardy space $H^{p}(\mathbb{D})$, is open (see Privalov [8] and Duren [2]):

How a function in $X$ can be recovered by the behavior of its Taylor coefficients?

Ideally, one would like to find a condition on the $\widehat{f}(n):=\frac{f^{n}(0)}{n !}$ (Taylor coefficient), which is both necessary and sufficient for $f$ to be in $X$ Of course, for $X=H^{p}, p=2$, the problem is completely solved: $f \in H^{2}$ if and only if $\sum_{n=0}^{\infty}|\widehat{f}(n)|^{2}<+\infty$. For $p=\infty$, the problem of coefficients was solved by I. Schur in 1919 (see, Privalov [ㅁ, Ch. 2]). Some classical results on the Taylor coefficients of functions in Hardy and Bergman spaces are also known (see, for instance, [2, 8, 14]). Some recent results about Taylor coefficients of $H^{1}$ functions and entire functions in the Fock spaces $F_{\alpha}^{p}$ have been obtained, respectively, by Pavlović [6] and Tung [13. But the general situation is much more complicated, and no complete answer is available (more informations about this are contained in [1] and references therein.)

Note that in his book [6], Pavlović proved the following characterization of functions belonging to the Hardy space $H^{1}$ :

$$
H^{1}=\left\{f \in \operatorname{Hol}(\mathbb{D}): \sup _{0<r<1} \frac{1}{2 \pi} \int_{0}^{2 \pi}\left|f\left(r e^{i t}\right)\right| d t<+\infty\right\} .
$$

Theorem 1.1. For a function $f$ analytic in $\mathbb{D}$, the following statements are equivalent:

(a) $f \in H^{1}$;

(b) $\sup _{n \geqslant 0} \frac{1}{a_{n}} \sum_{j=0}^{n} \frac{1}{j+1}\left\|S_{j}(f)\right\|_{H^{1}}<+\infty$;

(c) $\sup _{n \geqslant 0}\left\|P_{n} f\right\|_{H^{1}}<+\infty$.

Here $P_{n} f:=\frac{1}{a_{n}} \sum_{j=0}^{n} \frac{1}{j+1} S_{j}(f)$, where $a_{n}=\sum_{j=0}^{n} \frac{1}{j+1}, n=0,1,2, \ldots$, and $S_{j}(f)$ are the partial sums of the Taylor series for $f$. 
Popa [7] gave some interesting generalization of this result of Pavlović by proving a similar characterization of upper triangular trace class matrices. Recently, Karaev [3] gave some characterizations of Hardy and Besov classes of analytic functions on $\mathbb{D}$ with the variable exponents.

In this paper, we use the so-called Berezin symbols technique to characterize the Bers-type, Zygmund-type spaces, and the Bloch spaces $\mathcal{B}$ and $\mathcal{B}_{0}$. We also consider some spaces defined by the variable exponent. We discuss Toeplitz operators on the Bergman space $L_{a}^{2}(\mathbb{D})$

\section{BerEZin symbols And CHARACTERIZATIOns of the BERS-TyPe, Zygmund-Type} AND BLOCH SPACES

In this section, we characterize the spaces $H_{\alpha}^{\infty}, \mathcal{Z}^{\beta}, \mathcal{B}$ and $\mathcal{B}_{0}$ in terms of Berezin symbols of diagonal operators (associated with the Taylor coefficients of the functions from the spaces $H_{\alpha}^{\infty}, \mathcal{Z}^{\beta}, \mathcal{B}$ and $\left.\mathcal{B}_{0}\right)$ acting on the Hardy space $H^{2}=H^{2}(\mathbb{D})$.

Recall that Hardy-Hilbert space $H^{2}=H^{2}(\mathbb{D})$ is the collection of analytic functions in $\mathbb{D}$ which satisfy the inequality

$$
\|f\|_{H^{2}}:=\left(\sup _{0<r<1} \frac{1}{2 \pi} \int_{0}^{2 \pi}\left|f\left(r e^{i t}\right)\right|^{2} d \theta\right)^{\frac{1}{2}}<+\infty .
$$

It is well-known that $H^{2}$ is a reproducing kernel Hilbert space with the orthonormal basis $e_{n}(z)=z^{n}(n=0,1,2, \ldots)$, and consequently with the reproducing kernel

$$
k_{\lambda}(z)=\sum_{n=0}^{\infty} \overline{e_{n}(\lambda)} e_{n}(z)=\sum_{n=0}^{\infty} \bar{\lambda}^{n} z^{n}=\frac{1}{1-\bar{\lambda} z}, \lambda \in \mathbb{D} .
$$

The reproducing property means that $\left\langle f, k_{\lambda}\right\rangle_{H^{2}}=f(\lambda)$ for all $f \in H^{2}$ and $\lambda \in \mathbb{D}$. For any bounded sequence $\left(a_{n}\right)_{n \geqslant 0}$ of complex numbers $a_{n}$, we denote by $D_{\left(a_{n}\right)}$ the associate diagonal operator on the Hardy-Hilbert space $H^{2}$ which is defined by the formula

$$
D_{\left(a_{n}\right)} z^{n}=a_{n} z^{n}, \quad n=0,1,2, \ldots
$$

For any bounded linear operator $T$ on $H^{2}$, its Berezin symbol $\widetilde{T}$ is the following bounded complex-valued function in $\mathbb{D}$ :

$$
\widetilde{T}(\lambda):=\left\langle T \mathcal{K}_{\lambda}, \mathcal{K}_{\lambda}\right\rangle(\lambda \in \mathbb{D}),
$$

where

$$
\mathcal{K}_{\lambda}(z):=\frac{k_{\lambda}(z)}{\left\|k_{\lambda}(z)\right\|_{H^{2}}}=\left(1-|\lambda|^{2}\right)^{\frac{1}{2}}(1-\bar{\lambda} z)^{-1}
$$

is the normalized reproducing kernel of $H^{2}$, and $\operatorname{ber}(T):=\sup _{\lambda \in \mathbb{D}}|\widetilde{T}(\lambda)|$ is the Berezin number of $T$.

The following lemma is well-known.

Lemma 2.1. The Berezin symbol of the diagonal operator $D_{\left(a_{n}\right)}$ on the Hardy space $H^{2}$ is the following radial function:

$$
\widetilde{D}_{\left(a_{n}\right)}(|\lambda|)=\left(1-|\lambda|^{2}\right) \sum_{k=0}^{\infty} a_{k}|\lambda|^{2 k} \quad(\lambda \in \mathbb{D})
$$

Proof. Indeed, by using that

$$
k_{\lambda}(z)=\frac{1}{1-\bar{\lambda} z}=\sum_{k=0}^{\infty} \bar{\lambda}^{k} z^{k}
$$


is the reproducing kernel of $H^{2}$, we have:

$$
\begin{aligned}
\widetilde{D}_{\left(a_{n}\right)}(\lambda) & =\left\langle D_{\left(a_{n}\right)} \mathcal{K}_{\lambda}(z), \mathcal{K}_{\lambda}(z)\right\rangle=\left\langle D_{\left(a_{n}\right)} \frac{k_{\lambda}(z)}{\left\|k_{\lambda}(z)\right\|_{2}}, \frac{k_{\lambda}(z)}{\left\|k_{\lambda}(z)\right\|_{2}}\right\rangle \\
& =\left(1-|\lambda|^{2}\right)\left\langle D_{\left(a_{n}\right)} \sum_{k=0}^{\infty} \bar{\lambda}^{k} z^{k}, \sum_{k=0}^{\infty} \bar{\lambda}^{k} z^{k}\right\rangle=\left(1-|\lambda|^{2}\right)\left\langle\sum_{k=0}^{\infty} \bar{\lambda}^{k} a_{k} z^{k}, \sum_{k=0}^{\infty} \bar{\lambda}^{k} z^{k}\right\rangle \\
& =\left(1-|\lambda|^{2}\right) \sum_{k=0}^{\infty} a_{k}|\lambda|^{2 k} .
\end{aligned}
$$

Hence,

$$
\widetilde{D}_{\left(a_{n}\right)}(\lambda)=\left(1-|\lambda|^{2}\right) \sum_{k=0}^{\infty} a_{k}|\lambda|^{2 k}, \quad \lambda \in \mathbb{D}
$$

which proves formula (2).

Our next result characterizes the spaces $H_{\alpha}^{\infty}, \mathcal{Z}^{\beta}, \mathcal{B}$ and $\mathcal{B}_{0}$ in terms of behavior of the Berezin symbols of the corresponding diagonal operators mentioned above.

Theorem 2.1. Let a function $f(z)=\sum_{n=0}^{\infty} \widehat{f}(n) z^{n} \in \operatorname{Hol}(\mathbb{D})$ have a bounded sequence $\{\widehat{f}(n)\}_{n \geqslant 0}$ of Taylor coefficients $\widehat{f}(n)=\frac{f^{(n)}(0)}{n !},(n=0,1,2, \ldots)$. Then $f \in H_{\alpha}^{\infty}(\alpha \geqslant 0)$ if and only if

$$
\sup _{\substack{0 \leqslant r<1 \\ 0 \leqslant \theta<2 \pi}}(1-r)^{\alpha-1}\left|\widetilde{D}_{\left(\widehat{f}(n) e^{i n \theta}\right)}(\sqrt{r})\right|<+\infty .
$$

Proof. Indeed, let a function $f$ be as in the statement of the theorem. Then, rewriting $f$ and using Lemma 2.1, we obtain:

$$
\begin{aligned}
f(z) & =f\left(|z| e^{i \arg (z)}\right)=f\left(r e^{i \theta}\right)=\sum_{n=0}^{\infty} \widehat{f}(n)\left(r e^{i \theta}\right)^{n} \\
& =\sum_{n=0}^{\infty} \widehat{f}(n) e^{i n \theta} r^{n}=\frac{(1-r) \sum_{n=0}^{\infty} \widehat{f}(n) e^{i n \theta} r^{n}}{1-r}=\frac{\widetilde{D}_{\left(\widehat{f}(n) e^{i n \theta}\right)}(\sqrt{r})}{1-r} .
\end{aligned}
$$

Hence, for any $z=r e^{i \theta}$, with $r=|z|$ and $\theta=\arg (z), 0 \leqslant r<1,0 \leqslant \theta<2 \pi$, we have

$$
f(z)=\frac{\widetilde{D}_{\left(\widehat{f}(n) e^{i n \theta}\right)}(\sqrt{r})}{1-r} .
$$

Using (3), we get

$$
\left(1-|z|^{2}\right)^{\alpha}|f(z)|=\left(1-r^{2}\right)^{\alpha} \frac{\widetilde{D}_{\left(\widehat{f}(n) e^{i n \theta}\right)}(\sqrt{r})}{1-r}=(1-r)^{\alpha-1}(1+r)^{\alpha}\left|\widetilde{D}_{\left(\widehat{f}(n) e^{i n \theta}\right)}(\sqrt{r})\right|
$$

for all $r, 0 \leqslant r<1$ and all $\theta \in[0,2 \pi)$. Hence, we obtain

$$
(1-r)^{\alpha-1}\left|\widetilde{D}_{\left(\widehat{f}(n) e^{i n \theta}\right)}(\sqrt{r})\right| \leqslant\left(1-|z|^{2}\right)^{\alpha}|f(z)| \leqslant 2^{\alpha}(1-r)^{\alpha-1}\left|\widetilde{D}_{\left(\widehat{f}(n) e^{i n \theta}\right)}(\sqrt{r})\right|
$$

for all $z=r e^{i \theta} \in \mathbb{D}$. In particular, by (5) we conclude that that $f \in H_{\alpha}^{\infty}$ if and only if

$$
\sup _{\substack{0 \leqslant r<1 \\ \theta \in[0,2 \pi)}}(1-r)^{\alpha-1}\left|\widetilde{D}_{\left(\widehat{f}(n) e^{i n \theta}\right)}(\sqrt{r})\right|<+\infty .
$$


This completes the proof.

An immediate corollary of inequalities (5) is as follows.

Corollary 1. If $f \in H_{\alpha}^{p}$ and $\alpha>0$, then

$$
\sup _{\substack{0 \leqslant r<1 \\ 0 \leqslant \theta<2 \pi}}(1-r)^{\alpha-1}\left|\widetilde{D}_{\left(\widehat{f}(n) e^{i n \theta}\right)}(\sqrt{r})\right| \leqslant\|f\|_{p, \alpha} \leqslant 2^{\alpha} \sup _{\substack{0 \leqslant r<1 \\ 0 \leqslant \theta<2 \pi}}(1-r)^{\alpha-1}\left|\widetilde{D}_{\left(\widehat{f}(n) e^{i n \theta}\right)}(\sqrt{r})\right| .
$$

Theorem 2.2. Let a function $f(z)=\sum_{n=0}^{\infty} \widehat{f}(n) z^{n} \in \operatorname{Hol}(\mathbb{D})$ have the sequence $\{\widehat{f}(n)\}_{n \geqslant 0}$ of Taylor coefficients such that $\widehat{f}(n)=O\left(\frac{1}{n}\right)$ as $n \rightarrow \infty$. Then

(a) $f$ belongs to Bloch space $\mathcal{B}$ if and only if

$$
\sup _{\substack{0 \leqslant r<1 \\ 0 \leqslant \theta<2 \pi}}\left|\widetilde{D}_{\left((n+1) \widehat{f}(n+1) e^{i n \theta}\right)}(\sqrt{r})\right|<+\infty ;
$$

(b) $f \in \mathcal{B}_{0}$ if and only if

$$
\lim _{r \rightarrow 1}\left|\widetilde{D}_{\left((n+1) \widehat{f}(n+1) e^{i n \theta}\right)}(\sqrt{r})\right|=0
$$

for all $\theta \in[0,2 \pi)$.

Proof. It follows from the condition $\widehat{f}(n)=O\left(\frac{1}{n}\right)(n \rightarrow \infty)$ that $(n \widehat{f}(n))_{n}$ is bounded, and hence, the diagonal operator $D_{(n \widehat{f}(n))}$ is bounded on the Hardy-Hilbert space $H^{2}$. This implies immediately that $D_{\left((n+1) \widehat{f}(n+1) e^{i n \theta}\right)}$ is also bounded in $H^{2}$ for every fixed $\theta \in[0,2 \pi)$. Therefore, by formula (2) in Lemma 2.1, we have

$$
\begin{aligned}
\left(1-|z|^{2}\right) f^{\prime}(z) & =\left(1-r^{2}\right) \sum_{n=0}^{\infty} \widehat{f}(n) z^{n}=\left(1-r^{2}\right) \sum_{n=1}^{\infty} n \widehat{f}(n) z^{n-1} \\
& =\left(1-r^{2}\right) \sum_{n=0}^{\infty}(n+1) \widehat{f}(n+1) z^{n} \\
& =(1-r)(1+r) \sum_{n=0}^{\infty}(n+1) \widehat{f}(n+1) e^{i n \theta} r^{n} \\
& =(1+r)\left[(1-r) \sum_{n=0}^{\infty}\left((n+1) \widehat{f}(n+1) e^{i n \theta}\right) r^{n}\right] \\
& =(1+r) \widetilde{D}_{\left((n+1) \widehat{f}(n+1) e^{i n \theta}\right)}(\sqrt{r}) .
\end{aligned}
$$

Hence,

$$
\left(1-|z|^{2}\right) f^{\prime}(z)=(1+r) \widetilde{D}_{\left((n+1) \widehat{f}(n+1) e^{i n \theta}\right)}(\sqrt{r})
$$

for all $z=r e^{i \theta} \in \mathbb{D}$, where $r \in[0,1)$ and $\theta \in[0,2 \pi)$. Now formula (6) shows that $f \in \mathcal{B}$ if and only if

$$
\sup _{\substack{0 \leqslant r<1 \\ 0 \leqslant \theta<2 \pi}}\left|\widetilde{D}_{\left((n+1) \widehat{f}(n+1) e^{i n \theta}\right)}(\sqrt{r})\right|<+\infty
$$

and this proves (a).

On the other hand, it follows also immediately from (6) that $f \in \mathcal{B}_{0}$ if and only if

$$
\lim _{r \rightarrow 1}\left|\widetilde{D}_{\left((n+1) \widehat{f}(n+1) e^{i n \theta}\right)}(\sqrt{r})\right|=0
$$

for all $\theta, 0 \leqslant \theta<2 \pi$. This proves (b). 
Formula (6) implies also the following results.

Corollary 2. Assume that $f(z)=\sum_{n=0}^{\infty} \widehat{f}(n) z^{n}$ satisfies the condition of Theorem 2.2. If $f \in \mathcal{B}$, then

$$
\inf _{\substack{0 \leqslant r<1 \\ 0 \leqslant \theta<2 \pi}}\left|\widetilde{D}_{\left((n+1) \widehat{f}(n+1) e^{i n \theta}\right)}(\sqrt{r})\right|+|f(0)| \leqslant\|f\|_{\mathcal{B}} \leqslant|f(0)|+2 \sup _{\substack{0 \leqslant r<1 \\ 0 \leqslant \theta<2 \pi}}\left|\widetilde{D}_{\left((n+1) \widehat{f}(n+1) e^{i n \theta}\right)}(\sqrt{r})\right| .
$$

Corollary 3. If $f$ is the same as in Corollary 2, then we have:

(a) $\|f\|_{\mathcal{B}} \leqslant|f(0)|+2 \sup _{0 \leqslant \theta<2 \pi} \operatorname{ber}\left(D_{\left((n+1) \widehat{f}(n+1) e^{i n \theta}\right)}\right)$.

(b) $\|f\|_{\mathcal{B}} \leqslant|f(0)|+2 \sup _{n \geqslant 0}(n+1)|\widehat{f}(n+1)|$.

Clearly, Statement (a) implies Statement (b) since for each arbitrary fixed $\theta \in[0,2 \pi)$

$$
\operatorname{ber}\left(D_{\left((n+1) \widehat{f}(n+1) e^{i n \theta}\right)}\right) \leqslant\left\|D_{\left((n+1) \widehat{f}(n+1) e^{i n \theta}\right)}\right\|=\sup _{n \geqslant 0}(n+1)|\widehat{f}(n+1)| .
$$

Corollary 4. Each function $f(z)=\sum_{n=0}^{\infty} \widehat{f}(n) z^{n} \in \operatorname{Hol}(\mathbb{D})$ obeying $\widehat{f}(n)=o\left(\frac{1}{n}\right)$ as $n \rightarrow \infty$ belongs to the little Bloch space $\mathcal{B}_{0}$.

Proof. Indeed, since by assumption $\lim _{n \rightarrow \infty} n|\widehat{f}(n)|=0$, we see that $D_{\left((n+1) \widehat{f}(n+1) e^{i n \theta}\right)}$ is a compact diagonal operator, and since $H^{2}$ is the standard reproducing kernel Hilbert space (which means that the normalized reproducing kernel $\mathcal{K}_{\lambda}(z)=\frac{\left(1-|\lambda|^{2}\right)^{\frac{1}{2}}}{1-\bar{\lambda} z}$ weakly tends to zero as $\lambda$ approaches any boundary point of the unit disc $\mathbb{D}$ ), we obtain that $\lim _{r \rightarrow 1}\left|\widetilde{D}_{\left((n+1) \widehat{f}(n+1) e^{i n \theta}\right)}(\sqrt{r})\right|=0$ for all $\theta \in[0,2 \pi)$, which implies that $f \in \mathcal{B}_{0}$. The proof is complete.

Theorem 2.3. Let $\beta>0$ and $f(z)=\sum_{n=0}^{\infty} \widehat{f}(n) z^{n} \in \operatorname{Hol}(\mathbb{D})$ be a function such that $\widehat{f}(n)=O\left(\frac{1}{(n-1) n}\right)$ as $n \rightarrow \infty$. Then $f \in \mathcal{Z}^{\beta}$ if and only if

$$
\sup _{\substack{0 \leqslant r<1 \\ 0 \leqslant \theta<2 \pi}}(1-r)^{\beta-1}\left|\widetilde{D}_{\left((n+1)(n+2) \widehat{f}(n+2) e^{i n \theta}\right)}(\sqrt{r})\right|<+\infty .
$$

Proof. Since by assumption $((n-1) n \widehat{f}(n))_{n \geqslant 2}$ is a bounded sequence, the diagonal operator $D_{\left((n-1) n \widehat{f}(n) e^{i n \theta}\right)}$ is a bounded operator on the Hardy space $H^{2}$ for all $\theta \in[0,2 \pi)$. Then, by Lemma 2.1 we have:

$$
\begin{aligned}
\left(1-|z|^{2}\right)^{\beta}\left|f^{\prime \prime}(z)\right| & =\left(1-r^{2}\right)\left|\sum_{n=2}^{\infty}(n-1) n \widehat{f}(n) z^{n-2}\right| \\
& =\left(1-r^{2}\right)^{\beta}\left|\sum_{n=0}^{\infty}(n+1)(n+2) \widehat{f}(n+2) z^{n}\right| \\
& =\left(1-r^{2}\right)^{\beta}\left|\sum_{n=0}^{\infty}(n+1)(n+2) \widehat{f}(n+2) e^{i n \theta} r^{n}\right| \\
& =(1+r)^{\beta}(1-r)^{\beta-1}\left|(1-r) \sum_{n=0}^{\infty}\left[(n+1)(n+2) \widehat{f}(n+2) e^{i n \theta}\right] r^{n}\right|
\end{aligned}
$$




$$
=(1+r)^{\beta}(1-r)^{\beta-1}\left|\widetilde{D}_{\left((n+1)(n+2) \widehat{f}(n+2) e^{i n \theta}\right)} \sqrt{r}\right|
$$

and hence,

$$
\left(1-|z|^{2}\right)^{\beta}\left|f^{\prime \prime}(z)\right|=(1+r)^{\beta}(1-r)^{\beta-1}\left|\widetilde{D}_{\left((n+1)(n+2) \widehat{f}(n+2) e^{i n \theta}\right)}(\sqrt{r})\right|
$$

for all $z=r e^{i \theta} \in \mathbb{D}$. This equality shows that $f \in \mathcal{Z}^{\beta}$ if and only if

$$
\sup _{\substack{0 \leqslant r<1 \\ 0 \leqslant \theta<2 \pi}}(1-r)^{\beta-1}\left|\widetilde{D}_{\left((n+1) \widehat{f}(n+1) e^{i n \theta}\right)}(\sqrt{r})\right|<+\infty,
$$

which proves the theorem.

\section{Generalized subharmonicity and Toeplitz operators on Bergman space}

In this section we apply representations (4), (6) and (7) for studying the boundedness, compactness and belonging to Schatten-Neumann class for Toeplitz operators acting in the Bergman space $L_{a}^{2}=L_{a}^{2}(\mathbb{D})$.

Let $d A(z)$ be the area measure on $\mathbb{D}$ normalized so that the area of $\mathbb{D}$ is 1 . In terms of Cartesian and polar coordinates is reads as

$$
d A(z)=\frac{1}{\pi} d x d y=\frac{1}{\pi} r d r d \theta
$$

For $1 \leqslant p<+\infty$, the usual Lebesgue space $L^{p}(\mathbb{D}, d A)$ denote the Banach space of Lebesgue measurable functions $f$ on $\mathbb{D}$ with the norm

$$
\|f\|_{p}=\left[\int_{\mathbb{D}}|f(z)|^{p} d A(z)\right]^{\frac{1}{p}}<+\infty .
$$

The Bergman space $L_{a}^{p}=L_{a}^{p}(\mathbb{D})$ is defined to be the subspace of $L^{p}(\mathbb{D}, d A)$ consisting of analytic functions. For $p=2, L_{a}^{2}$ is a reproducing kernel Hilbert space with the reproducing kernel

$$
k(z, w)=\frac{1}{(1-z \bar{w})^{2}}
$$

Recall that $P: L^{2}(\mathbb{D}, d A) \rightarrow L_{a}^{2}$ is the Bergman projection and this is an integral operator given by the formula

$$
\operatorname{Pf}(z)=\int_{\mathbb{D}} k(z, w) f(w) d A(w)=\int_{\mathbb{D}} \frac{f(w)}{(1-z \bar{w})^{2}} d A(w) .
$$

Given a function $\varphi \in L^{\infty}(\mathbb{D})$, we define the Toeplitz operator $T_{\varphi}$ on $L_{a}^{2}$ by $T \varphi f=P(\varphi f)$, $f \in L_{a}^{2}$. Since the Bergman projection has the unit norm, we clearly get $\left\|T_{\varphi}\right\| \leqslant\|\varphi\|_{\infty}$. More information about Bergman space Toeplitz operators can be found, for example, in the book by K.Zhu [14].

Definition 3.1 ([14]). Suppose $f$ is a nonnegative function on $\mathbb{D}$. We say that $f$ has a generalized subharmonic property if there exists a constant $C>0$ such that

$$
f(z) \leqslant \frac{C}{|D(z, r)|} \int_{D(z, r)} f(w) d A(w)
$$

for all $z \in \mathbb{D}$. Here for each $r>0$ and $a \in \mathbb{D}$

$$
D(a, r):=\{z \in \mathbb{D}: \beta(z, a)<r\}
$$


is the Bergman disc with the Bergman metric

$$
\beta(z, w)=\frac{1}{2} \log \frac{1+\rho(z, w)}{1-\rho(z, w)},
$$

where $\rho(z, w)=\left|\frac{z-w}{1-\bar{z} w}\right|(z, w \in \mathbb{D})$ is the pseudo-hyperbolic distance on $\left.\mathbb{D}\right)$ and $|D(a, r)|$ is the normalized area of $D(a, r)$.

Before proving a next theorem, we mention the following known result [14].

Lemma 3.1. If $\varphi \geqslant 0$ possesses the generalized subharmonic property, then

(1) $T_{\varphi}$ is bounded on $L_{a}^{2}$ if and only if $\varphi$ is bounded as $|z| \rightarrow 1^{-}$;

(2) $T_{\varphi}$ is compact on $L_{a}^{2}$ if and only if $\varphi(z) \rightarrow 0$ as $|z| \rightarrow 1^{-}$;

(3) $T_{\varphi}$ is in $S_{p}\left(L_{a}^{2}\right)$ if and only if $\varphi \in L^{p}(\mathbb{D}, d \lambda)$, where $d \lambda(z)=\frac{d A(z)}{\left(1-|z|^{2}\right)^{2}}$ is the Möbius invariant measure on $\mathbb{D}$.

Theorem 3.1. Let a function $f(z)=\sum_{n=0}^{\infty} \widehat{f}(n) z^{n} \in \operatorname{Hol}(\mathbb{D})$ have a bounded sequence $\{\widehat{f}(n)\}_{n \geqslant 0}$ of Taylor coefficients. Let $\alpha \geqslant 0$. Then

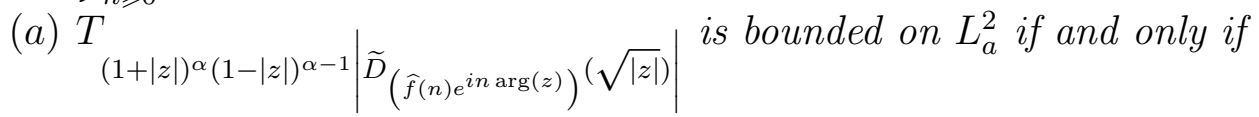

$$
(1-|z|)^{\alpha-1}\left|\widetilde{D}_{\left(\widehat{f}(n) e^{i n \arg (z)}\right)}(\sqrt{|z|})\right|
$$

is bounded as $|z| \rightarrow 1^{-}$;

(b) $T_{(1+|z|)^{\alpha}(1-|z|)^{\alpha-1}} \mid \widetilde{D}_{\left(\widehat{f}^{(n) e^{i n} \arg (z)}\right)}\left(\sqrt{|z|)} \mid\right.$ is compact on $L_{a}^{2}$ if and only if

$$
(1-|z|)^{\alpha-1}\left|\widetilde{D}_{\left(\widehat{f}(n) e^{i n \arg (z)}\right)}(\sqrt{|z|})\right| \rightarrow 0
$$

as $|z| \rightarrow 1^{-}$;

(c) $T$ belongs to the Schatten-Neumann class $S_{p}:=S_{p}\left(L_{a}^{2}\right)$ if and only if

$$
\int_{\mathbb{D}}(1+|z|)^{\alpha p}(1-|z|)^{p(\alpha-1)}\left|\widetilde{D}_{\left(\widehat{f}(n) e^{i n \arg (z)}\right)}(\sqrt{|z|})\right|^{p} d_{\lambda}(z)<+\infty
$$

where $d_{\lambda}(z)=\frac{d A(z)}{(1-|z|)^{2}}$ is the Möbius invariant measure on $\mathbb{D}$.

Proof. Indeed, one can prove that if $f$ is analytic, $p>0$, and $\alpha$ is real, then the function $(1-|z|)^{\alpha}|f(z)|^{p}$ possesses the generalized subharmonic property (see K.Zhu [14]). In particular, the function $\left(1-|z|^{2}\right)^{\alpha}|f(z)|$ possesses the same property. On the other hand, by formula (4),

$$
\left(1-|z|^{2}\right)^{\alpha}|f(z)|=(1+|z|)^{\alpha}(1-|z|)^{\alpha-1}\left|\widetilde{D}_{\left(\widehat{f}(n) e^{i n \arg (z)}\right)}(\sqrt{|z|})\right|
$$

for all $z \in \mathbb{D}$. Now the statement of theorem is immediately implied by Lemma 3.1 .

By using formulae (6) and (7), the nextg two results can be proved by the same method as in the above proof, and therefore the proof is omitted.

Theorem 3.2. Let a function $f(z)=\sum_{n=0}^{\infty} \widehat{f}(n) z^{n} \in \operatorname{Hol}(\mathbb{D})$ have the sequence $\{\widehat{f}(n)\}_{n \geqslant 0}$ of Taylor coefficients such that $\widehat{f}(n)=O\left(\frac{1}{n}\right)$ as $n \rightarrow \infty$. Then

(a) $T_{\left(1-|z|^{2}\right)\left|f^{\prime}(z)\right|}$ is bounded on $L_{a}^{2}$ if and only if $\mid \widetilde{D}_{\left((n+1) \widehat{f}(n+1) e^{i n \arg (z)}\right)}(\sqrt{|z|)} \mid$ is bounded as $|z| \rightarrow 1^{-}$ 
(b) $T_{\left(1-|z|^{2}\right)\left|f^{\prime}(z)\right|}$ is compact on $L_{a}^{2}$ if and only if

$$
\left|\widetilde{D}_{\left((n+1) \widehat{f}(n+1) e^{i n \arg (z)}\right)}(\sqrt{|z|})\right| \rightarrow 0
$$

as $|z| \rightarrow 1^{-}$

(c) $T_{\left(1-|z|^{2}\right)\left|f^{\prime}(z)\right|}$ is in $S_{p}\left(L_{a}^{2}\right)$ if and only if

$$
\int_{\mathbb{D}}(1+|z|)^{p}\left|\widetilde{D}_{\left((n+1) \widehat{f}(n+1) e^{i n \arg (z)}\right)}(\sqrt{|z|})\right|^{p} d_{\lambda}(z)<+\infty .
$$

Theorem 3.3. Let $\beta>0$ and $f(z)=\sum_{n=0}^{\infty} \widehat{f}(n) z^{n} \in \operatorname{Hol}(\mathbb{D})$ be a function such that $\widehat{f}(n)=O\left(((n-1) n)^{-1}\right)$ as $n \rightarrow \infty$. Then

(a) $T_{\left(1-|z|^{2}\right)^{\beta}\left|f^{\prime \prime}(z)\right|}$ is bounded on $L_{a}^{2}$ if and only if $(1-|z|)^{\beta-1}\left|\widetilde{D}_{\left((n+1)(n+2) \widehat{f}(n+2) e^{i n \arg (z)}\right)}(\sqrt{|z|})\right|$ is bounded as $|z| \rightarrow 1^{-}$;

(b) $T_{\left(1-|z|^{2}\right)^{\beta}\left|f^{\prime \prime}(z)\right|}$ is compact on $L_{a}^{2}$ if and only if

$$
(1-|z|)^{\beta-1}\left|\widetilde{D}_{\left((n+1)(n+2) \widehat{f}(n+2) e^{i n \arg (z)}\right)}(\sqrt{|z|})\right| \rightarrow 0
$$

as $|z| \rightarrow 1^{-}$

(c) $T_{\left(1-|z|^{2}\right)^{\beta}\left|f^{\prime \prime}(z)\right|}$ is in $S_{p}\left(L_{a}^{2}\right)$ if and only if

$$
(1+|z|)^{\beta}(1-|z|)^{\beta-1}\left|\widetilde{D}_{\left((n+1)(n+2) \widehat{f}(n+2) e^{i n \arg (z)}\right)}(\sqrt{|z|})\right| \in L^{p}(\mathbb{D}, d \lambda) .
$$

\section{Characterization of MiXed NORM SPACE $H_{p(t), q(t), \gamma(t)}$ With VARIABle EXPONEnts}

Let $\mathbb{T}=\partial \mathbb{D}$ and let $p=p(t)$ and $q(t), t \in \mathbb{T}$, be bounded positive measurable functions defined on $\mathbb{T}$, and let $\gamma(t)>-1$ on $\mathbb{T}$. Following by Kokilashvili and Paatashvili [4, 5] (see also Karaev [2]), we say that the analytic function $f$ in $\mathbb{D}$ belongs to the Hardy class $H^{p(t)}$ if

$$
\sup _{0<r<1} \frac{1}{2 \pi} \int_{0}^{2 \pi}\left|f\left(r e^{i t}\right)\right|^{p(t)} d t<+\infty
$$

where $p(t)=p\left(e^{i t}\right), t \in[0,2 \pi)$.

Similarly, we say that a function $f \in \operatorname{Hol}(\mathbb{D})$ belongs to the mixed norm space $H_{p(t), q(t), \gamma(t)}$ with the variable exponents if

$$
\|f\|_{H_{p(t), q(t), \gamma(t)}^{q(t)}}:=\int_{0}^{1}\left(\frac{1}{2 \pi} \int_{0}^{2 \pi}\left|f\left(r e^{i t}\right)\right|^{p(t)} d t\right)^{\frac{q(t)}{p(t)}}(1-r)^{\gamma(t)} d r<+\infty,
$$

where $p(t)=p\left(e^{i t}\right), q(t)=q\left(e^{i t}\right)$ and $\gamma(t)=\gamma\left(e^{i t}\right), t \in[0,2 \pi)$.

For $p(t)=p=$ const $>0, q(t)=q=$ const $>0$ and $\gamma(t)=\gamma=$ const $>-1$, the class $H_{p(\cdot), q(\cdot), \gamma(\cdot)}$ coincides with the class $H_{p, q, \gamma}$.

The following theorem characterize the spaces $H_{p(\cdot), q(\cdot), \gamma(\cdot)}$ in terms of Berezin symbols and Taylor coefficients. 
Theorem 4.1. Let $f(z)=\sum_{n=0}^{\infty} \widehat{f}(n) z^{n} \in \operatorname{Hol}(\mathbb{D})$ be a function with the bounded sequence

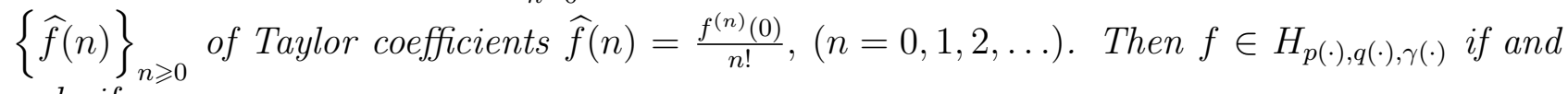
only if

$$
\int_{0}^{1}\left(\frac{1}{2 \pi} \int_{0}^{2 \pi}\left|\widetilde{D}_{\left(\widehat{f}(n) e^{i n t}\right)}(\sqrt{r})\right|^{p(t)} d t\right)^{\frac{q(t)}{p(t)}}(1-r)^{\frac{\gamma(t) p(t)-q(t)}{p(t)}} d r<+\infty
$$

Proof. Using formula (2) in Lemma 2.1, we rewrite the function $f(z)=\sum_{n=0}^{\infty} \widehat{f}(n) z^{n}$ as follows:

$$
f(z)=f\left(r e^{i t}\right)=\frac{\widetilde{D}_{\left(\widehat{f}(n) e^{i n t}\right)}(\sqrt{r})}{1-r}
$$

for each $z=r e^{i t} \in \mathbb{D}$. Now the statement of the theorem follows the definition of the space $H_{p(\cdot), q(\cdot), \gamma(\cdot)}$. Namely, in view of $(8)$, we obtain

$$
\begin{aligned}
\int_{0}^{1}\left(\frac{1}{2 \pi} \int_{0}^{2 \pi}\left|f\left(r e^{i t}\right)\right|^{p(t)} d t\right)^{\frac{q(t)}{p(t)}}(1-r)^{\gamma(t)} d r & =\int_{0}^{1}\left(\frac{1}{2 \pi} \int_{0}^{2 \pi}\left|\frac{\widetilde{D}_{\left(\widehat{f}(n) e^{i n t}\right)}(\sqrt{r})}{1-r}\right|^{p(t)} d t\right)^{\frac{q(t)}{p(t)}}(1-r)^{\gamma(t)} d r \\
& =\int_{0}^{1}\left(\frac{1}{2 \pi} \int_{0}^{2 \pi}\left|\widetilde{D}_{\left(\widehat{f}(n) e^{i n t}\right)}(\sqrt{r})\right|^{p(t)} d t\right)^{\frac{q(t)}{p(t)}}(1-r)^{\gamma(t)-\frac{q(t)}{p(t)}} d r .
\end{aligned}
$$

This yields that $f \in H_{p(\cdot), q(\cdot), \gamma(\cdot)}$ if and only if

$$
\int_{0}^{1}\left(\frac{1}{2 \pi} \int_{0}^{2 \pi}\left|\widetilde{D}_{\left(\widehat{f}(n) e^{i n t}\right)}(\sqrt{r})\right|^{p(t)} d t\right)^{\frac{q(t)}{p(t)}}(1-r)^{\gamma(t)-\frac{q(t)}{p(t)}} d r<+\infty .
$$

The proof is complete.

\section{REFERENCES}

1. J.M. Ash, M.T. Karaev. On the boundary behavior of special classes of $C^{\infty}$-functions and analytic functions // Intern. Math. Forum. 7:1-4, 153-166 (2012).

2. P. Duren. Theory of $H^{p}$ spaces. Dover Publications, Mineola (2000).

3. M.T. Karaev. A characterization of the some function classes // J. Funct. Spaces. Appl. 2012, id 796798 (2012).

4. V. Kokilashvili and V. Paatashvili. On Hardy classes of analytic functions with a variable exponent // Proc. A. Razmadze Math. Inst. 142:1, 134-137 (2006).

5. V. Kokilashvili and V. Paatashvili. On the convergence of sequences of functions in Hardy classes with a variable exponent // Proc. A. Razmadze Math. Inst. 146:1, 124-126 (2008).

6. M. Pavlović. Introduction to Function Spaces on the Disk. Matematicki Institut SANU, Belgrade (2004).

7. N. Popa. A characterization of upper triangular trace class matrices // Compt. Rend. Math. 347:1-2, 59-62 (2009).

8. I.I. Privalov. Granicnye svoistva analiticeskih funkciü. GosTekhIzdat, Moscow (1950) (in Russian).

9. Y. Ren. New criteria for generalized weighted composition operators from mixed norm spaces into Zygmund-type spaces // Filomat. 26:6, 1171-1178 (2012). 
10. J. Shi. Inequalities for the integral means of holomorphic functions and their derivatives in the unit ball of $\mathbb{C}^{n} / /$ Trans, Amer. Math. Soc. 328:2, 619-637 (1991).

11. S. Stević. Weighted composition operators between mixed norm spaces and $H_{\alpha}^{\infty}$ spaces in the unit ball // J. Ineq. Appl. 2007:1, 1-9 (2008).

12. S. Stević. Norm and essential norm of composition followed by differentiation from $\alpha-B l o c h$ spaces to $H_{\mu}^{\infty} / /$ Appl. Math. Comput. 207:1, 225-229 (2009).

13. J. Tung. Taylor coefficients of functions in Fock spaces // J. Math. Anal. Appl. 318:2, 397-409 (2006).

14. K. Zhu, Operator theory in function spaces. Marcel, New York (1990).

15. K. Zhu. Bloch type spaces of analytic functions // Rocky Mount. J. Math. 23:3, 1143-1177 (1993).

16. X. Zhu, Generalized weighted composition operators from Bloch spaces into Bers- type spaces // Filomat. 26:6, 1163-1169 (2012).

Mubariz Garayev,

Department of Mathematics, College of Science,

King Saud University,

P.O. Box 2455, Riyadh 11451, Saudi Arabia

E-mail: mgarayev@ksu.edu.sa

Hocine Guediri,

Department of Mathematics, College of Science,

King Saud University,

P.O. Box 2455, Riyadh 11451, Saudi Arabia

E-mail: hguediri@ksu.edu.sa

Houcine Sadraoui,

Department of Mathematics, College of Science,

King Saud University,

P.O. Box 2455, Riyadh 11451, Saudi Arabia

E-mail: sadrawi@ksu.edu.sa 